\title{
Primary Package Identifier
}

National Cancer Institute

\section{Source}

National Cancer Institute. Primary Package Identifier. NCI Thesaurus. Code C101699.

The main identifier of a package type. 